\title{
COMO ESTUDAR A "FAMILIA" E O "PAI"?
}

\section{Maria Auxiliadora Dessen' Charlie Lewis}

RESUMO - Este artigo aborda algumas questões teóricas, metodológicas e práticas sobre temas relativos à influência paterna e à natureza das interações paicriança e, também, algumas dimensões que as pesquisas sobre "famflia" e "pai" deveriam considerar, no Brasil. A discussão será baseada nos pressupostos de que: a) há vários tipos de famílias; b) os pais desempenham papéis diferentes em diferentes épocas e contextos culturais; $c$ ) os papéis paternos são multidimensionais e complexos. Para compreender o que é ser "pai" é preciso, primeiro, conhecer o que acontece dentro da "família", levando em consideração as interações e as relações desenvolvidas entre os seus diferentes subsistemas, bem como o contexto social no qual as "famílias" estão inseridas.

Palavras-Chave: famila, pai, pesquisa, psicologia, relações pai-filho.

\section{HOW CAN WE UNDERSTAND FAMIIIES AND FATHERS?}

ABSTRACT - We will discuss some theoretical, methodological, and practical issues in current research focusing on the nature of father-child interactions and paternal influence. Moreover, we will suggest some dimensions which research on families and fathers in Brazil should consider. This discussion will be based on the following assumptions: there are several kinds of families; fathers play different roles in different cultural contexts and historical times; and paternal roles are multidimensional and complex. To understand "fathers" we have to know firstly what happens within the "family", taking into account the interactions and relationships developed between the different family subsystems as well as the social context in which that families are embedded.

Key Words: family, fathers, research, psychology, father-child relations.

Este artigo tem como objetivo principal discutir alguns aspectos teóricos, metodológicos e práticos baseados na suposição de que as análises contemporâneas de "famílias" têm sido demasiadamente limitadas em seus focos. Para isto, serão comparadas as literaturas sobre "família" e "pai", as quais

1 Endereço para correspondência: Maria Auxiliadora Dessen. Laboratório de Desenvolvimento Familiar - IP - Universidade de Brasilia. Campus Universitário - Asa Norte - Brasília - DF CEP 70910-900 . Fone (061) 3072625 (R 409/417); e-mail dessen@unb.br

${ }^{2}$ Universidade de Lancaster

Paidéia, FFCLRP-USP, Rib. Preto, Fev/Ago 98. 
têm sido tratadas, até agora, de forma distinta. Nós começaremos discutindo, brevemente, o conceito de "família" na pesquisa contemporânea e também algumas questões teóricas e metodológicas destacadas por pesquisadores da área de psicologia da família. Na segunda parte deste artigo, focalizaremos as concepções de "pai" e seus papéis dentro da "família", para, em seguida, discutirmos alguns aspectos práticos sobre a implementação de pesquisa nesta área.

Nós esperamos que esta discussão tenha um impacto sobre os planejamentos de pesquisa no Brasil, quer aumentando a quantidade de estudos que incluam a "família" como uma unidade de análise, incorporando conseqüentemente o "pai" em suas amostras, quer focalizando variáveis culturais importantes para a compreensão do desenvolvimento humano. Devido à enormidade desta tarefa, nossa intenção não é apresentar uma revisão da literatura corrente sobre "família" e "pai", mas simplesmente apontar alguns tópicos que consideramos relevantes para a investigação de tais temas.

\section{Questões Teóricas e Metodológicas em Psicologia da Família}

\subsection{Questões conceituais}

Não há dúvida de que tem havido mudanças acentuadas na nossa compreensão do desenvolvimento familiar (ex.: Cusinato, 1996) e que não podemos mais continuar caracterizando a família como uma simples unidade nuclear tradicional. Por exemplo, Hodkin, Vacheresse e Buffet (1996), ao estudarem o conceito de família, mostraram que diferentes indivíduos incluíam em suas famílias, além dos membros nucleares, as seguintes categorias: namorado(a), avô, avó, amigos intimos e animais de estimação. Estes autores mostraram também que, apesar de o agrupamento nuclear ser considerado central na maioria dos estudos, a metodologia usada na pesquisa afeta o relato das pessoas sobre quem, de fato, eles consideram como membros de sua família. Por exemplo, quando eles aplicaram a tarefa de "Resposta-Livre" (os próprios sujeitos geram suas listas de membros familiares) depois de uma tarefa de "Checklist" (Listas para Assinalar), um número maior de pessoas foi incluído na "família". Portanto, parece que "quem" é considerado parte da família depende, sobretudo, da maneira como os pesquisadores fazem esta pergunta em seus estudos.

Também não é surpreendente o fato de que os indivíduos descrevem a família de forma complexa. Por muitas décadas, os cientistas sociais vêm apontando que a unidade nuclear ( 2 genitores casados possuindo crianças) é, de fato, a forma minoritária de família (Rapoport, Rapoport \& Strellitz, 1976). 
Assim, como afirma Petzold (1996, p. 25), não é mais possivel usar o conceito tradicional de família em pesquisa científica". Enquanto a unidade nuclear "moderna", aquela composta por genitores casados ou por um único genitor morando com seus filhos não casados em uma mesma casa (Petzold, 1996, p. 29), representa uma pequena maioria, formas alternativas sempre existiram e continuam aumentando, tais como casais homossexuais, casais amasiados e famílias sucessivas, incluindo aquelas recasadas e/ou em que todos os membros moram juntos (ex.: Cusinato, 1996; Lamb, 1997; Phares, 1996; Tasker \& Golombok, 1997).

Como Petzold (1996) sugere, uma definição científica de família necessita levar em consideração a pluralidade de formas atuais de família. Sua "abordagem ecopsicologica" parece ser apropriada para compreender todos os tipos de familias porque ela inclui uma ampla gama de formas de vida familiar, isto é, não somente as diferentes formas tradicionais como também as novas formas alternativas. A definição de família, de acordo com este modelo, é baseada nas relações íntimas e intergeracionais, incorporando variáveis externas, características das relações de esposa ou não-esposa e das relações genitores-criança, bem como outras pessoas que podem fazer parte da família.

De acordo com o modelo ecopsicológico, há 196 formas possíveis de família, as quais são o resultado da combinação de 14 variáveis que contribuem para a caracterização da família atual. Por exemplo, casamento legal ou coabitação, rendimentos separados ou compartilhados, relacionamento com pessoas consanguíneas ou por casamento, casais com ou sem criança(s), genitores ou padrasto/madrasta, desempenho de papéis subordinados/dominantes ou igualitários. É possível selecionar todos estes tipos de família em um único projeto de pesquisa? É óbvio que não. Entretanto, é muito importante definir o tipo de unidade social que estamos estudando, a fim de obter um corpo de conhecimento mais sistematizado sobre cada um deles. Além disto, as pesquisas futuras em psicologia da família deveriam levar em consideração estas várias formas de vida familiar em seus planejamentos.

\subsection{Tipos e níveis de análises}

Tão importante quanto uma definição adequada de família é o uso de conceitos apropriados de interação e relação social que reflitam o processo interacional e não simplesmente o comportamento de indivíduos, separadamente. Portanto, é fundamental que tenhamos conceitos formais e claros de interações e relações sociais, como aqueles propostos por Hinde $(1979 ; 1997)$. Seus conceitos expressam a necessidade de considerar as relações diádicas, triádicas e poliádicas entre os vários subsistemas familiares (Dessen, 1994). 
Neste contexto, o pai é somente um membro do sistema e seus papéis precisam ser investigados de acordo com as interações e as relações desenvolvidas entre as diferentes constelações que compõem a família.

Assim, seria muito útil descrever as dimensões de conteúdo, qualidade e padrões de interacões, bem como alguns aspectos das relações tais como reciprocidade, complementaridade, percepção interpessoal, compromisso e intimidade, ao focalizarmos os papéis de pai em um determinado estudo. Em sua maioria, os pesquisadores têm criado categorias analíticas que não medem, de fato, o processo interacional entre genitores-criança, pelo menos de acordo com os níveis definidos por Hinde (1997). Além disso, a tendência para descrever aspectos das interações envolvendo apenas alguns dos membros familiares pode explicar algumas das inconsistências encontradas nas pesquisas sobre o papel do pai, que foram destacadas por Lewis (1997).

Como as relações interpessoais envolvem pessoas com características individuais e histórias distintas de relacionamentos, que estão inseridas dentro de uma rede social específica, em um determinado contexto cultural (Hinde, 1997), muitas são as variáveis interligadas que afetam o curso destas relações ao longo da vida. Assim, os pesquisadores deveriam considerar estas estreitas conexões entre as variáveis, implementando projetos complexos que permitissem capturar, também, as mudanças sobre o tempo. Devido à complexidade da rede de relações familiares, o desenvolvimento individual não pode ser visto isolado do desenvolvimento da família enquanto grupo. Portanto, a família deveria ser considerada também como um grupo que tem seu próprio curso evolutivo, tendo, conseqüentemente, que realizar várias tarefas de desenvolvimento (Kreppner, 1989; 1991).

Neste contexto, duas perspectivas têm particularmente contribuído para orientar as pesquisas: teoria dos sistemas familiares (Minuchin, 1990) e teoria do sistema ecológico de Bronfenbrenner (Bronfenbrenner, 1986; 1992). A família é conceituada pela teoria dos sistemas familiares como uma série de sistemas interligados, envolvendo o indivíduo, a família e o ambiente familiar. Segundo Mann (1996), a maior contribuição desta perspectiva é ver a familia como um sistema adaptável, capaz de promover o equilíbrio entre uma tendência homeostática ou auto-reguladora e uma capacidade para transformação; sobretudo, a perspectiva dos sistemas focaliza as mudanças na família (p. 480). A abordagem dos sistemas para o desenvolvimento da criança considera, portanto, as mudanças e o desenvolvimento associados às mudanças que ocorrem no ciclo de vida individual, dentro de um dado contexto familiar e social. 


\section{3. $\mathrm{Q}$ sistema social mais ample}

A família não pode ser examinada como um sistema isolado. A teoria do sistema ecológico de Bronfenbrenner $(1986 ; 1992)$ orienta os pesquisadores no sentido de efetuar conexões mais amplas entre o sistema familiar e os demais. Em geral, esta teoria focaliza o indivíduo em relação às circunstâncias de sua vida, tanto físicas como sociais. Ela leva em consideração não somente as características individuais e as relações dentro da família, como também as mudanças no ambiente próximo (mesosistema) e no contexto social mais amplo (macrosistema), em um determinado tempo histórico.

Kaslow (1996) apresenta várias razões para a necessidade de se incluir os contextos econômico e político na pesquisa sobre família. Segundo esta autora, para muitas famílias, especialmente para aquelas de países como Peru, Haiti, Rússia e Somália, a vida é muito complicada e freqüentemente não previsível. Estas famílias experienciam uma sensação de não ter controle sobre suas vidas porque conflitos políticos expolsivos causam turbulência em suas vidas pessoais (p. 128). Há vários problemas sociais que estão sendo associados a contextos econômicos e políticos por pesquisadores, especialmente em países como o Brasil. Assim, seria prudência, por exemplo, negligenciar as circunstâncias sociais e as interconexões entre os vários sistemas e subsistemas sociais ao estudarmos as famílias brasileiras de crianças em situação de rua. Dessen e Biasoli-Alves (1997) propuseram a implementação de estudos sobre "famílias" que vivem em condições desfavoráveis como uma alternativa para ajudar as organizaçóes governamentais e não-governamentais na busca por soluções de problemas sociais.

Até o presente momento, nós temos enfatizado a complexidade do termo família, a dinâmica das interações e relações, a natureza interligada das trajetórias de vida de cada membro e as influências sociais sobre estas trajetórias. Se nossas análises estiverem corretas (e nós estamos sendo breves neste artigo), há sérias implicações para os planejamentos de pesquisa atuais e futuros. No tópico a seguir, tentaremos demonstrar algumas destas complexidades, focalizando o pai como um dos membros da família. Ainda, argumentaremos que a compreensão dos papéis masculinos em diferentes tipos de coabitação pode clarificar muito do que é conhecido, hoje, sobre a família, além de abrir importantes dimensões de investigação para os pesquisadores.

\section{Como os Pais se Ajustam às Famílias?}

\subsection{Questões conceituais}


Em outro artigo (Lewis \& Dessen, 1998), nós fornecemos algumas evidências a respeito da variedade de formas de participação paterna dentro da vida familiar que, por sua vez, podem ter diferentes determinantes e conseqüências (ver, por exemplo, Barnett \& Baruch, 1988; Lewis, 1997). Mediante tal diversidade de participação e determinantes, nós nos perguntamos: o que significa ser um pai? Ou, na verdade, o que é um pai? A resposta a esta questão, com base na pesquisa contemporânea, revela algumas das complexidades de nossas definições de família. Uma única criança pode ter um pai biológico, alguém que provê a sua subsistência (um pai econômico), um homem (ou homens) que ela identifica como uma figura de pai (um pai social) e alguém que é legalmente identificado como um pai. Um homem pode desempenhar todos estes papéis, embora cada um possa ser desempenhado por diferentes homens (ver Marsiglio, 1995, para uma discussão). Se adicionarmos a estas perspectivas os pontos de vista de cada membro familiar sobre os conceitos de família e pai, a definição torna-se ainda mais complexa.

A paternidade como uma atividade deve ser vista em uma perspectiva cultural e histórica. Segundo Fein (1978) e Schwebel, Fine e Moreland (1988), os pais têm adotado um dos seguintes papéis: tradicional, moderno ou emergente. $O$ primeiro refere-se aos pais cujas atividades primárias centram-se ao redor do mundo do trabalho, tendo eles pouco a fazer em relação às atividades de cuidar de suas crianças (p. 299). Por outro lado, os pais que desempenham um papel moderno vêem o desenvolvimento bem sucedido da criança como um objetivo importante, especialmente nas áreas de desenvolvimento da identidade do papel sexual, desempenho acadêmico e desenvolvimento moral (p. 299). Já, o papel emergente refere-se ao pai que participa mais igualmente com sua esposa das atividades de cuidados da criança (p. 299). Como muitos outros, estes autores acham que o papel de pai emergente pode favorecer o desenvolvimento positivo da criança e também enriquecer as relações familiares. Entretanto, a idéia de uma simples progressão histórica destes papéis tem sido criticada. Por exemplo, Lewis e O'Brien (1987) sugerem que há muitos comentários sociais a respeito das mudanças nos papéis paternos, mas poucas evidências que os apoiam. Portanto, é necessário implementar mais estudos que adotem uma perspectiva de pesquisa cultural, a fim de verificar se, de fato, tem ocorrido mudanças nestes papéis.

Para compreender o que significa ser um pai em famílias contemporâneas, é muito importante responder as seguintes questões: o que os pais e suas crianças realmente fazem juntos (tipos de atividade)? Quais são os determinantes da paternidade e seus impactos sobre o papel do pai? É também importante compreender o processo de se tornar pai, bem como a influência paterna na vida familiar, especialmente no desenvolvimento da criança. Neste artigo, o 
nosso objetivo é apenas enfatizar como todos os tipos de pais listados acima se ajustam à nossa compreensão de família.

\subsection{Quais informantes e quais relações deveriam ser focalizadas?}

Nas pesquisas sobre famílias, a mãe e a criança têm sido as fontes de informação mais importantes, enquanto o pai é, ainda, freqüentemente considerado através do relato das mães (Bronstein \& Cowan, 1988; Phares, 1996). Isto tem ocorrido, a despeito da crença generalizada de que a melhor fonte de informação sobre o comportamento ou as atitudes dos pais é o próprio pai. Embora os planejamentos de pesquisa estejam incluindo cada vez mais o relato dos próprios pais, a maioria deles tem como objetivo comparar as diferenças entre os comportamentos de mães e pais (Bronstein \& Cowan, 1988), visando dar subsídios à oposição aos próprios direitos à paternidade (Bronstein, 1988). É necessário lidar diretamente com pais, mães e crianças se o objetivo for medir as diferenças entre eles e os processos pelos quais os indivíduos constroem as relações familiares. Entretanto, os pesquisadores não deveriam ficar demasiadamente preocupados com a correspondência nos relatos ou classificações de todos eles, especialmente quando se tem como objetivo a compreensão do assunto investigado sob a perspectiva da famíla como grupo.

Como afirma Phares (1996), uma vez que as relações estudadas são, em sua maioria, diádicas, particularmente as interações mãe-criança, as análises não fornecem informações contrastando as influências maternas e paternas. Se ela (e nossa análise na primeira parte deste artigo) estiver correta, torna-se necessário, também, compreender os vários níveis de envolvimento paternal porque cada genitor fornece contribuições únicas para a criança no curso de seu desenvolvimento. Portanto, é importante que as pesquisas se tornem mais sensíveis aos tipos de relação paterna que a criança está exposta. Isto teria dois efeitos: primeiro, capacitar-nos-ia a explorar, empiricamente, as interações e relações com base nos níveis teóricos propostos por Hinde (ver secção 1.2., acima); segundo, ajudar-nos-ia a compreender melhor os papéis dentro das famílias. Por exemplo, é necessário compreender o que acontece às crianças que vivem em famílias cuja relação entre os diferentes tipos de pais (ex.: social, legal, biológico) é complexa. No Reino Unido e nos Estados Unidos, muitas pesquisas têm focalizado grupos complexos como famílias recasadas (aquelas cujo marido e esposa trazem consigo os filhos de outros casamentos) e famílias com crianças produzidas por novas tecnologias, tais como fertilização in vitro, porque estes tipos de modos de vida acentuam as tensões entre os diferentes tipos de família.

Em síntese, diferentes informantes fornecem diferentes informações; 
portanto, o ideal seria obter dados a partir de múltiplos informantes porque dados de um único indivíduo não podem refletir facilmente as diferentes perspectivas sobre o assunto investigado (Phares, 1996). Se, ainda, considerarmos a necessidade de estudar outros membros familiares e outras pessoas fora da família, tais como amigos e vizinhos, nós enfrentaremos um grande desafio metodológico relacionado, especialmente, às medidas de desenvolvimento de relações (ver secção 3). Na verdade, os informantes deveriam ser selecionados de acordo com o tipo de perspectiva que se quer explorar mais profundamente e não, simplesmente, para verificar evidências conflitantes.

\subsection{O pai. a família e os processos sociais em pesquisa}

As análises da participação e envolvimento de pais, baseadas na adoção de uma perspectiva do sistema social mais amplo, levantam questōes sobre as complexidades dos tipos de coabitação familiar (ver secção 1.3). Para conhecer a famíla mais profundamente, nós deveríamos, primeiro, ampliar os grupos de pesquisa incluindo pesquisadores de diferentes disciplinas, como sociologia e antropologia. Revisando a literatura, Pillemer e McCartney (1991) encontraram várias linhas de pesquisas sobre as relações genitores-criança sendo conduzidas, mas com pouca interação entre os participantes de diferentes áreas. Para eles, a comunicação entre psicólogos e sociólogos é ainda limitada em relação a este assunto, embora algum progresso tenha sido alcançado recentemente.

Na verdade, alguns livros (ex.: Bronstein \& Cowan, 1988; Lewis \& O'Brien, 1987; McKee \& O'Brien, 1982; Pillemer \& McCartney, 1991) têm ressaltado a necessidade de uma abordagem interdisciplinar para o estudo da família e do pai. Os colaboradores destes volumes são de diversos campos: psicologia, sociologia, psiquiatria, pediatria, direito, trabalho social, enfermagem, entre outros. Estes livros parecem ter sido organizados com os objetivos de acentuar pontos de comparação e contraste entre os diferentes campos e ajudar aqueles que estão trabalhando sozinhos em seus projetos. A nossa suposição é de que somente uma abordagem interdisciplinar poderia ajudar a compreender o tópico, embora, na prática, pesquisas "verdadeiramente" interdisciplinares sejam difíceis de implementar e analisar.

\section{Questões Práticas em Pesquisa}

Já que somos obrigados a enfrentar a necessidade de desenvolver pesquisa em contextos culturais complexos, quais são os aspectos que devemos prestar particular atenção? Nesta secção, discutiremos seis questões que consideramos importantes: utilizar a diversidade cultural para estudar famílias 
e pais em ação, estar sensível à consequiente complexidade de pesquisas comparativas, estudar a família como um grupo, usar planejamentos longitudinais e abordagens multimetodológicas, ser imaginativo ao planejar pesquisa e refletir sobre a complexidade dos planejamentos.

\subsection{Utilizar a diversidade cultural para estudar as famílias e os pais em}

ação

Pesquisadores de diferentes comunidades (e culturas) deveriam unirse em projetos comuns com a finalidade de coletar dados usando a mesma metodologia, ao mesmo tempo. A maior parte das pesquisas culturais compara dados com base em duas culturas; poucos estudos envolvem três ou mais contextos culturais (ex.: Tudge, Hogan, Tammevesky, Kulokova, Meltsas, Snezhkova \& Putnam, 1997). Na verdade, pouquíssimas pesquisas têm como objetivo comparar diferentes grupos culturais. Este tipo de abordagem é essencial para promover avanços na área da psicologia da família e tem sido evidenciada nas tendências de pesquisas recentes (ex.: Bornstein, 1991). Entretanto, há uma série de dificuldades na implementação de projetos desta natureza, especialmente relacionadas a custos financeiros e interpretação de dados.

3.2. Estar sensível à conseqüente complexidade de pesquisas comparativas

Quando um planejamento transcultural é empregado, é muito importante que a coleta de dados seja conduzida por pesquisadores nativos das culturas representadas no estudo. Segundo Wang, Mylander e Goldin-Meadow (1996), os nativos de uma determinada cultura conhecem as normas daquela cultura, o que poderia favorecer o desenvolvimento de interações culturalmente apropriadas entre pesquisadores e sujeitos: o pesquisador nativo compreende as sutilezas de uma interação e, provavelmente, está mais capacitado para clarificar questöes e dúvidas no momento em que elas surgem (p. 373).

\subsection{Estudar a família como um todo: a questão dos níveis de análise}

Se nossas suposições a respeito da necessidade de se estudar a variedade de interações e relações dentro de uma família estiverem corretas, faz-se necessário focalizar os vários membros familiares. $O$ uso de múltiplos informantes em pesquisa requer a tomada de decisão sobre a adequação ou não de se agregar ou separar os dados obtidos. Phares (1996) afirma que ambas as estratégias têm vantagens e desvantagens e os pesquisadores precisam 
considerar como cada uma delas influencia seus próprios projetos de pesquisa (p. 270). Portanto, os pesquisadores devem ter não somente estas questões em mente, mas também considerar, na prática, a necesssidade de investigar todos os subsistemas familiares, incluindo as relações diádicas, triádicas e poliádicas desenvolvidas entre os diferentes membros da família. É perfeitamente possível imaginar como seria difícil analisar todas as relações diádicas, triádicas e poliádicas desenvolvidas entre todos os membros de uma família composta por quatro pessoas.

Além disso, temos que nos defrontar com o fato de que a psicologia não tem ainda uma linguagem apropriada para descrever o desenvolvimento de relações sociais (Cowan, 1988; Hinde, 1997) o que poderia, inclusive, favorecer comparações entre estudos transculturais. Pesquisadores da área de desenvolvimento social têm tentado diferentes sistemas, mas uma linguagem especializada tende a isolar os grupos de pesquisa da comunidade mais ampla (mesmo outros pesquisadores), além do que, uma linguagem vernacular tende a ser interpretada de diferentes modos e, portanto, a ser mal compreendida.

Nós gostaríamos, ainda, de ressaltar dois aspectos a respeito das medidas que construímos quando focalizamos as relações sociais. O primeiro diz respeito ao esforço que deveríamos fazer para desenvolver uma linguagem comum, apropriada para descrever o desenvolvimento das relações familiares, criando instrumentos adequados para a coleta de dados, tanto dentro como fora da família. Com o desenvolvimento de categorias mais complexas, nós poderíamos nos aproximar da compreensão das complexidades das famílias. Segundo, é necessário reconhecer o fato de que tanto os dados coletados quanto a linguagem e a teoria que desenvolvemos são limitados por um conjunto de suposições que os pesquisadores e as famílias estudadas trazem consigo e que, de alguma maneira, influenciam o processo de investigação. Na literatura sobre pais, por exemplo, há uma série de experimentos (ver detalhes em Lewis, 1997) sugerindo que as diferenças observadas no comportamento de mães e pais podem ser o produto de suas diferentes reações ao processo de ser observado, mais do que de suas diferenças sexuais.

\subsection{Usar planejamentos longitudinais e abordagens multimetodológicas}

Além das questões relativas à interdisciplinaridade, estudos transculturais e níveis de análise em pesquisa, é importante ter em mente que o método de coleta de dados desempenha um papel crucial na determinação da qualidade dos resultados de pesquisa. Por exemplo, o planejamento longitudinal é o mais apropriado quando a principal variável de estudo é o desenvolvimento humano. A despeito das dificuldades práticas inerentes ao uso deste 
tipo de planejamento, tais como alto custo financeiro e freqüentes "atritos" entre participantes, os pesquisadores deveriam perseguir tais objetivos ao planejarem seus estudos. Há vários pequenos cortes que poderiam ser feitos em pesquisas desta natureza, aproveitando amostras já estudadas em pesquisas transversais ou usando planejamentos de natureza mista, combinando procedimentos transversais e longitudinais.

\subsection{Ser imaginativo ao planejar sua pesquisa}

É preciso reconhecer a necessidade de construir uma diversidade de planejamentos de pesquisa se desejamos, de fato, compreender a família e os papéis paternos mais claramente. Não é suficiente, simplesmente, assumir que planejamentos longitudinais e dados culturalmente exóticos resolverão todos os problemas. É nossa responsabilidade encorajar a próxima geração de pesquisadores a ser criativa em relação às maneiras de explorar as complexidades dos sistemas e das relações familiares. Qualquer revisão da literatura mostrará uma homogeneidade "não brilhante" em muitos estudos. Por exemplo, planejamentos envolvendo diferentes tipos de famílias são mais comuns do que planejamentos intrafamiliares (ex.: Bronstein \& Cowan, 1988; Cusinato, 1996; Pillemer \& McCartney, 1991), apesar de os primeiros serem considerados interessantes somente para examinar variáveis cruciais que distinguem tais "tipos" de família. Além disto, esta abordagem é limitada, uma vez que algumas questões só podem ser compreendidas se examinadas as condições dentro da família e as diferenças entre os membros familiares. Portanto, planejamentos intrafamiliares também contribuem e deveriam estar sendo mais empregados.

Em um dos projetos atuais de Lewis, o objetivo é explorar como os pais se ajustam à vida familiar, em coabitações onde há uma criança em idade escolar (11-16 anos), e quais aspectos são transmitidos de uma geração para outra. Neste projeto, envolvendo cada membro da família, têm sido aplicadas entrevistas detalhadas, incluindo questões "abertas", cuja tarefa do respondente é expressar seus próprios pontos de vista sobre um determinado tópico e também a sua opinião ou "suposição" a respeito dos pontos de vista dos outros membros familiares em relação ao mesmo tópico. Este procedimento possibilita não somente compreender a maneira como os indivíduos apresentam a família em uma entrevista, mas também identificar os padrões de consistência e inconsistência de cada membro familiar em relação a si próprio e entre aqueles que moram juntos.

Outras mudanças mais simples poderiam ser feitas. Por exemplo, a maioria dos estudos sobre a família e o pai tem dependido de entrevistas e 
questionários padronizados, quando outros métodos deveriam ser considerados juntamente com estes procedimentos fáceis e de baixo custo financeiro. A observação direta, dada a sofisticação da tecnologia de vídeo e de registro de eventos, é uma excelente alternativa já que possibilita desenvolver medidas sistemáticas para descrever e avaliar as relações familiares, especialmente em um nível micro. Enquanto nos anos 70 e 80, alguns pesquisadores estavam preocupados com as diferenças entre os dados obtidos através dos procedimentos de entrevista e observação, hoje, os pesquisadores relacionam ambos os procedimentos como compatíveis. Há suficientes exemplos na literatura empírica mostrando que tais técnicas podem ser usadas de uma maneira complementar. A pesquisa de Rutter (1989), sobre crianças criadas sob a custódia de autoridades locais, ilustra bem este fato. Nesta pesquisa, foram usadas observações e entrevistas detalhadas, mostrando que os indivíduos prognosticados por meio de entrevistas como sendo prováveis de encontrar dificuldade no desempenho da paternidade foram, também, observados (por classificadores "ingênuos") interagindo de um modo menos harmonioso com seus bebês.

\subsection{Refletir sobre a complexidade de seu planejamento de pesquisa}

Quando vários membros familiares estão envolvidos, é preciso considerar díades, tríades e tétrades (ex.: Kreppner, Paulsen \& Schuetze, 1982) e mesmo a família como "um grupo". Que diferenças podem ser esperadas quando se está coletando dados sobre cada um destes níveis? As pesquisas que permitiriam responder esta questão não são ainda conclusivas. Por exemplo, os dados de Lamb (1977) sugerem que a presença da mãe em interações triádicas envolvendo a criança decresce a qualidade da interação pai-criança. $O$ fato de que a interação pai-criança é mais frequientemente triádica do que diádica (com a mãe sendo, invariavelmente, a base de comparação) tem levado à interpretação de que os pais são menos responsivos do que as mães (para uma discussão detalhada, ver Lewis, 1997). Similarmente, alguns estudos têm sugerido que as mães também podem ser menos responsivas na presença de pais (ex.: Dessen, 1992). Tais descobertas simplesmente destacam a necessidade de mais pesquisas sobre os "efeitos reativos" da presença de outros membros nas interações familiares. Alguns dados sugerem que pares pai-filha são mais influenciados pela presença de um observador do que outras díades genitor-criança (Lewis, Kier, Hyder, Prenderville, Pullen \& Stephens, 1996), mas tais descobertas apenas arranham a superfície da complexidade das medidas de interação familiar. Alguns fatores como o sexo do pesquisador tem sido demasiadamente negligenciados.

Nesta secção, nós tentamos fornecer alguns exemplos de dimensões/ 
questões que permeiam os numerosos modos de investigar os tópicos relacionados a família e ao pai. Considerar todos os membros da família, usando métodos variados de coleta de dados e análises qualitativa e/ou quantitativa, constitui um enorme desafio para o pesquisador. Se, além disto, nós acrescentarmos as dimensões de planejamento longitudinal, uso de amostras representativas e, também, coleta de dados com os mesmos instrumentos (ou adaptados), o processo de pesquisa poderia parecer mais um pesadelo do que algo agradável. Entretanto, noites em claro são, freqüentemente, um pré-requesito para se fazer uma boa pesquisa. Como afirma Phares (1996): não é somente de mais pesquisas que necessitamos, mas sim de pesquisas mais relevantes, que sejam baseadas em vários contextos étnicos e sócio-econômicos e que aumentem a validade ecológica dos estudos sobre o pai (p. 313).

\section{Considerações Finais}

Se nós estivermos corretos em assumir que estudar a diversidade cultural constitui uma rota apropriada para tornar as teorias mais compreensíveis e melhorar as pesquisas sobre família, o Brasil seria considerado um laboratório ideal. As diferenças nos estilos de vida entre as suas diferentes comunidades parecem ser um recurso excelente, especialmente se tais diferenças forem consideradas em relação ao tempo histórico e ao contexto sócio-econômico. Estudar a diversidade cultural poderá nos ajudar a construir não somente um conceito mais válido sobre famflia, mas também perspectivas teóricas centradas nos micro e macrosistemas do desenvolvimento familiar. Nós precisamos investigar quais são, de fato, as influências sobre as famílias brasileiras. Alguns tipos de dados (por exemplo, papéis maternos e paternos, práticas educativas) ajudam a identificar fatores de risco no desenvolvimento da família e da criança e sugerem que nós deveríamos explorar o contexto sócioeconômico de tais influências (Biasoli-Alves \& Zamberlan, 1996), particularmente em tempos de mudanças social e econômica.

Mudanças culturais e sociais necessitam ser adicionadas a estas perspectivas. No Reino Unido, por exemplo, tem havido um aumento acentuado de divórcio nos últimos 30 anos, de tal forma que é provável que as crianças estejam vivendo tanto em lares separados como em lares intactos. Marin (1996) investigou os conceitos de família e a percepção de problemas familiares em crianças de 4 a 8 anos, de diferentes cidades do norte da Itália, que viviam em lares cujas famílias estavam experienciando circunstâncias maritais críticas. Mesmo assim, estas crianças definiram a família como um grupo de pessoas que moram juntas sob o mesmo teto; pessoas que se tornaram cada vez 
mais unidas, com o passar do tempo, pelos laços biológicos (p. 432). Portanto, somos obrigados a reconhecer as diversidades e as influências culturais, a fim de explorar os valores e as crenças sobre como as crianças deveriam ser tratadas dentro do contexto familiar (ex.: Tudge e cols, 1997).

O que nós sabemos sobre o papel do pai brasileiro? Que tipo de relação há entre estes pais e suas crianças? $O$ que pais e crianças fazem juntos em situações rotineiras? Estes assuntos têm recebido pouquíssima atenção no Brasil; portanto, seria uma boa idéia se os pesquisadores interessados em estudar as relações genitores-criança, em diferentes pontos do ciclo de desenvolvimento humano, pudessem se unir a nós neste esforço para compreender os papéis do pai brasileiro. Nós esperamos que este artigo traga contribuições para os pesquisadores que investigam questões sobre as "famílias brasileiras", especialmente no que tange a gerar novas idéias para pesquisa.

\section{Referências Bibliográficas}

Barnett, R.C. \& Baruch, G.K. (1988). Correlates of fathers' participation in family work. Em P. Bronstein \& C.P. Cowan (Orgs.), Fatherhood today: Men's changing role in the family (pp. 66-78). New York: Wiley.

Biasoli-Alves, Z.M.M. \& Zamberlan, M.A. (1996). Demographic characteristics and childrearing practices of low socioeconomic status families. Em M. Cusinato (Org.), Research on family resources and needs across the world (pp. 199-216). Milano: LED.

Bornstein, M. H. (Org.) (1991). Cultural approaches to parenting. Hillsdale, N.J.: Erlbaum.

Bronfenbrenner, U. (1986). Ecology of the family as a context for human development: Research perspectives. Developmental Psychology, 22, 723742.

Bronfenbrenner, U. (1992). Ecological system theory. Em R. Vasta (Org.), Six theories of child development - Revised formulations and current issues (pp. 187-249). London: JKP.

Bronstein, P. (1988). Father-child interaction: Implications for gender role socialization. Em P. Bronstein \& C.P. Cowan (Orgs.), Fatherhood today: men's changing role in the family (pp. 107-124). New York: Wiley.

Bronstein, P. \& Cowan, C.P. (Orgs.) (1988). Fatherhood today: Men's changing role in the family. New York: Wiley.

Cowan, P.A. (1988). Becoming a father: A time of change. An opportunity for 118 Paidéia, FFCLRP-USP, Rib. Preto, Fev/Ago 98. 
development. Em P. Bronstein \& C.P. Cowan (Orgs.), Fatherhood today: men's changing role in the family (pp. 13-35). New York: Wiley.

Cusinato, M. (Org.) (1996). Research on family resources and needs across the world. Milano: LED.

Dessen, M.A. (1992). Efeitos do nascimento de uma segunda criança no comportamento e nas relações entre o primogênito e os genitores. Tese de Doutorado, Instituto de Psicologia, Universidade de São Paulo, São Paulo.

Dessen, M.A. (1994). Interações e relações no contexto familiar: questões teóricas e metodológicas. Psicologia: Teoria e Pesquisa, 10 (2), 213220.

Dessen, M.A. \& Biasoli-Alves, Z.M.M. (1997, novembro). O estudo da familia como base para a promoção da tolerância. Trabalho apresentado no Seminário Internacional: Ciência, Cientistas e a Tolerância. São Paulo: UNESCO e USP.

Fein, R.A. (1978). Research on fathering: Social policy and an emergent perspective. Journal of Social Issues, 34(1), 122-135.

Hinde, R.A. (1979). Towards understanding relationships. London: Academic Press.

Hinde, R.A. (1997). Relationships: A dialectical perspective. Hove: Psychological Press.

Hodkin, B.; Vacheresse, A. \& Buffet, S. (1996). Concept of family: Methodological issues in assessing perceived family memberships. Em M. Cusinato (Org.), Research on family resources and needs across the world (pp. 45-54). Milano: LED.

Kaslow, F.K. (1996). Families and family psychology in the 21st century: Recent trends and predictions for the future. Em M. Cusinato (Org.), Research on family resources and needs across the world (pp. 127-144). Milano: LED.

Kreppner, K. (1989). The interplay between individual and family development: Some results from a 7-year longitudinal study. Em M.A. Luszcz \& T. Nettelbeck (Orgs.), Psychological development: perspectives across the life-span (pp. 25-36). North-Holland: Elsevier.

Kreppner, K. (1991). Observation and longitudinal approach in infancy research. Em M.E. Lamb \& H. Keller (Orgs.), Infant development: perspectives from german-speaking countries (pp. 151-178). Hillsdale, N.J.: Erlbaum. 
Kreppner, K.; Paulsen, S. \& Schuetze, Y. (1982). Infant and family development: From triads to tetrads. Human Development, 25, 373-391.

Lamb, M. (1977). Infant social cognition and "second-order" effects. Infant Behaviour and Development, 1(1), 1-10.

Lamb, M. (Org.) (1997). The role of the father in child development. New York: Wiley.

Lewis, C. (1997). Fathers and preschoolers. Em M. Lamb (Org.), The role of the father in child development (pp. 121-142). New York: Wiley.

Lewis, C. \& Dessen, M.A. (1998, maio). O pai no contexto familiar. Trabalho apresentado no VII Simpósio da Associação Nacional de Pesquisa e Pós-Graduação em Psicologia. Gramado: ANPEPP

Lewis, C.; Kier, C.; Hyder, C.; Prenderville, N.; Pullen, J. \& Stephens, A. (1996). Observer influences on fathers and mothers: An experimental manipulation of the structure and function of parent-infant conversation. Early Development and Parenting, 5(1), 57-68.

Lewis, C. \& O'Brien, M. (1987). Reassessing fatherhood. London: Sage

Mann, C. (1996). Girls's talk: An adolescent perspective on personal relationships within the changing family. Em M. Cusinato (Org.), Research on family resources and needs across the world (pp. 477-490). Milano: LED.

Marin, M.L. (1996). The concept of family and the perception of family problems in developmental age children, with particular reference to critical marriage situations. Em M. Cusinato (Org.), Research on family resources and needs across the world (pp. 413-434). Milano: LED.

Marsiglio, W. (1995). Artificial reproduction and paternity testing. Implications for fathers. Em M.C.P. van Donger, G.A.B. Frinking \& J.G. Jacobs (Orgs.), Changing fatherhood. An interdisciplinary perspective (pp. 159-178). Amsterdam: Thesis Publishers.

Mckee, L. \& O'Brien, M. (1982). The father figure. London: Tavistock.

Minuchin, P. (1990). A systems perspective on development. Em R. Hinde \& J. Stevenson-Hinde (Orgs.), Relationships within families (pp. 7-26). New York: Oxford University Press.

Petzold, M. (1996). The psychological definition of "the family". Em M. Cusinato (Org.), Research on family resources and needs across the world (pp. 25-44). Milano: LED. 
Phares, V. (1996). Fathers and developmental psychopathology. New York: Wiley.

Pillemer, K. \& McCartney, K. (Orgs.) (1991). Parent-child relations throughout life. Hillsdale, N.J.: Erlbaum.

Rapoport, R.; Rapoport, R.N. \& Strellitz, Z. (1976). Fathers, mothers and others. London: Routledge and Kegan Paul.

Rutter, M. (1989). Pathways from childhood to adult life. Journal of Child Psychology and Psychiatry, 30, 23-51.

Schwebel, A.I.; Fine, M.\& Moreland, J.R. (1988). Clinical work with divorced and widowed fathers: the adjusting family model. Em P. Bronstein \& C.P. Cowan (Orgs.), Fatherhood today: men's changing role in the family (pp. 299-319). New York: Wiley.

Tasker, F. \& Golombok, S. (1997). Growing up in a lesbian family. New York: Guilford.

Tudge, J.; Hogan, D.; Tammeveski, P.; Kulokova, N.; Meltsas, M.; Snezhkova, I. \& Putnam, S. (1997, july). Social change, socio-economic status, and the development of self-direction in children: a comparison of Russia, Estonia, and the United States. Trabalho apresentado no V Congresso Europeu de Psicologia, Dublin.

Wang, X.L.; Mylander, C. \& Goldin-Meadow, S. (1996). A cross-cultural study of mothers' interaction in Chinese and American families. Em M. Cusinato (Org.), Research on family resources and needs across the world (pp. 25-44). Milano: LED. 